\title{
Conus infarction after non-guided transcoccygeal ganglion impar block using particulate steroid for chronic coccydynia
}

\author{
Dorothy Khai Chin Kuek ${ }^{1} \cdot$ Siok Li Chung ${ }^{2} \cdot$ Umme Sara Zishan $^{3}$ - Joseph Papanikitas ${ }^{2} \cdot$ Sarah Yanny $^{2}$. \\ Tom Meagher ${ }^{2} \cdot$ James Teh ${ }^{4} \cdot$ Richard Hughes $^{2} \cdot$ Wei Chuen Liong ${ }^{2} \cdot$ David McKean $^{2}$
}

Received: 9 July 2019 / Revised: 23 October 2019 / Accepted: 25 October 2019

(c) International Spinal Cord Society 2019

\begin{abstract}
Introduction Ganglion impar block (GIB) is a well-recognised treatment for chronic coccydynia. Several side effects have previously been described with this procedure, including transient motor dysfunction, bowel, bladder, and sexual dysfunction, neuritis, rectal perforation, impingement of the sciatic nerve, cauda equina syndrome, and infection.

Case presentation We describe the first report of imaging-documented conus infarction after an unguided-GIB performed in theatre using particulate steroids for a 17-year-old patient with coccydynia. Immediately post-GIB, patient developed transient neurological deficits in her lower limbs of inability to mobilise her legs that lasted for $24 \mathrm{~h}$. These include back and leg pain, decreased power and movement, increased tone, brisk reflexes, reduced light touch sensation and proprioception of legs up to the T10 level. Urgent MRI spine showed intramedullary hyperintense signal within the conus and mild restricted diffusion on the distal cord and conus, suggestive of an acute conus infarction. On follow-up, the GIB did not result in symptom improvement of coccydynia and there was persistent altered sensation of her legs.

Discussion Various approaches of ganglion impar block have been described and performed in the past with different imaging techniques and injectants. A few cases of unusual neurological complications have been reported with the use of epidural steroid injections and ganglion impar block. Clinicians should be aware of the possible neurological complications following ganglion impar blocks and the risk of inadvertent intravascular injection of particulate steroids can potentially to be minimised by using imaging guidance.
\end{abstract}

\section{Introduction}

Over the past century, sympathetic ganglion blocks are increasingly being used for controlling a variety of painful conditions. These include coccygodynia, complex regional pain syndrome, cancer pain, and postherpetic neuralgia [1]. Some non-painful conditions such as post-traumatic stress disorder and hyperhidrosis can also be treated by sympathetic

David McKean

david.mckean@nhs.net

1 Derriford Hospital, University Hospitals Plymouth NHS Trust, Plymouth, UK

2 Stoke Mandeville Hospital, Buckinghamshire Healthcare NHS Trust, Aylesbury, UK

3 Queen Elizabeth University Hospital NHSGGC, Glasgow, UK

4 Nuffield Orthopaedic Centre, Oxford University Hospitals NHS Trust, Oxford, UK blocks [1]. Ganglion impar block (GIB) is a common type of ganglion block, which blocks the ganglion impar or ganglion of Walther. Ganglion impar is a solitary retroperitoneal structure forming the tail of the bilateral sympathetic chain, it is situated anteriorly to the coccyx and supplies nociceptive and sympathetic fibres to the perineal region $[2,3]$. Intractable coccygeal and perineal or pelvic pain has been successfully treated by GIB [1]. A number of papers have described the potential for rare complications, including motor, bowel, bladder, and sexual dysfunction, neuritis, rectal perforation, impingement of the sciatic nerve, cauda equina syndrome, and infection. However, there is a paucity of complications that have actually been reported [4].

Spinal cord infarction is a rare but potentially devastating event, which has been described following a number of percutaneous spinal interventions such as transforaminal epidural injections in the cervical, lumbar, or caudal regions $[5,6]$. While such complications have typically been associated with the use of particulate steroid solutions, one case of conus medullaris infarction was reported in 2016 
following a right L4 transforaminal epidural steroid injection using dexamethasone [6]. One previous case of transient sensory loss with involvement of bladder and bowel control following fluoroscopic-guided GIB was described in 2017 , with resolution of the neurological symptoms on follow-up [7]. Here, we present the first documented case of conus medullaris infarction following unguided transsacrococcygeal GIB for the treatment of a young patient with chronic coccydynia and no previously known neurology.

\section{Case presentation}

A 17-year-old female presented with a background of severe coccydynia secondary to falling heavily off a slide at the age of 13. Previous sacrococcygeal MRI demonstrated a significantly increased intercoccygeal angle, a small bursa at the dorsal aspect of the coccyx, and a prominent draining vein at the ventral aspect of the coccyx. There was no evidence of tethered spinal cord, low thecal sac or low-lying conus. Her conus medullaris was confirmed to be of normal position at the level of T12 vertebra. Neurological examinations at the time of presentation were normal.

The patient underwent a conventional non-guided transsacrococcygeal GIB. The procedure was performed in theatre under conscious sedation with intravenous midazolam, for diagnostic and therapeutic purposes. Four millilitres of $0.5 \%$ Bupivacaine and $1 \mathrm{ml}$ of $40 \mathrm{mg}$ Kenalog (Triamcinolone acetonide) was injected through the sacrococcygeal joint with a 23-gauge (blue) needle using anatomical guidance.

In the immediate post-procedural period, while in recovery, the patient reported inability to mobilise her lower limbs. She denied any urinary disturbance, sickness, or neck stiffness at the time. On examination, all pulses were present with a normal upper limb neurological exam. However, her lower limb neurological exam showed increased tone with bilateral-positive clonus (five beats), and a significant decrease in power bilaterally to MRC 1 in L2/L3 myotomes and MRC 0 in L4/L5/S1 myotomes. This was accompanied by a decrease in light touch sensation and a decreased proprioception to the T10 level. Brisk ankle and knee reflexes were noted in both legs. Severe back pain radiating to her legs was also reported. Upper limb neurological examination was normal. Cardiovascular, respiratory, and abdominal examination were unremarkable. Peripheral pulses within the lower limbs were also normal. There was a high index of clinical suspicion of neurological complication and an urgent MRI of the spine was arranged. This demonstrated intramedullary hyperintense signal within the conus on sagittal STIR and T2 images and axial T2weighted images (Figs. 1 and 2a, b). There was mild restricted diffusion on the corresponding DWI/ADC images of the distal cord and conus. The MRI appearances were consistent with an acute conus infarction (Fig. 3).

The patient was admitted and over the following $24 \mathrm{~h}$ spontaneously recovered motor function. However, altered sensation persisted.

At clinic follow-up at 6 months, the patient reported ongoing tingling and heavy feeling in legs and subjective weakness. Examination revealed an increased tone in her left leg, bilateral crossed adductors, normal power, bilateral ankle clonus, down going plantar reflexes, no ataxia, a negative Romberg's test and a negative Gower's test. Unfortunately, she reported no improvement in her symptoms of coccygeal pain.

\section{Discussion}

Coccydynia can be debilitating, treatment options include corticosteroid injections into the sacrococcygeal junction, caudal epidural space, or blocking the Ganglion Impar by injecting local anaesthetic agents [8]. Sympathetic ganglia inputs are involved in various complex pain pathophysiology, these inputs are suggested to cause the loss of regular inhibitory influence on pain [1]. Therefore, ganglion blocks can block these sympathetic neurons and decrease central hyperexcitability [1]. Although rare, reported complications of GIB include misplaced injectate into the nerve roots causing numbness, paraesthesia, or neuritis [7].

There are various approaches to performing a GIB. The first approach was described in 1990 by Plancarte et al. [9] as a needle insertion through the anococcygeal ligament until reaching anteriorly to the sacrococcygeal joint. Later on, transsacrococcygeal approach, other intercoccygeal joint approaches and paracoccygeal approaches were described $[8,10]$. Transsacrococcygeal approach was first described by Wemm and Saberski in 1995 and is recommended for its direct approach with a shorter needle path, hence less expertise is needed to perform this approach $[1,3]$. Transsacrococcygeal approach of GIB has been claimed to be generally effective and safe in the management of coccyx and perineal pain according to prospective evaluation, but more randomised controlled trials with larger sample sizes are needed to conclude on its efficacy $[1,3]$.

Imaging guidance can include fluoroscopy and more recently $\mathrm{CT}$, ultrasonography, and MR imaging guidance [11-13]. A fluoroscopic-guided transsacrococcygeal approach is much preferred by clinicians in comparison to a conventional anatomical technique, as it is technically more feasible and has a lower risk of visceral injuries [14]. The needle used for GIB can be curved, or a needle-insideneedle coaxial technique might be required if the sacrococcygeal disc is ossified later in life [3, 14]. 
The selection of agents for GIB include local anaesthetics and steroids as used in this case or neurolytic agents

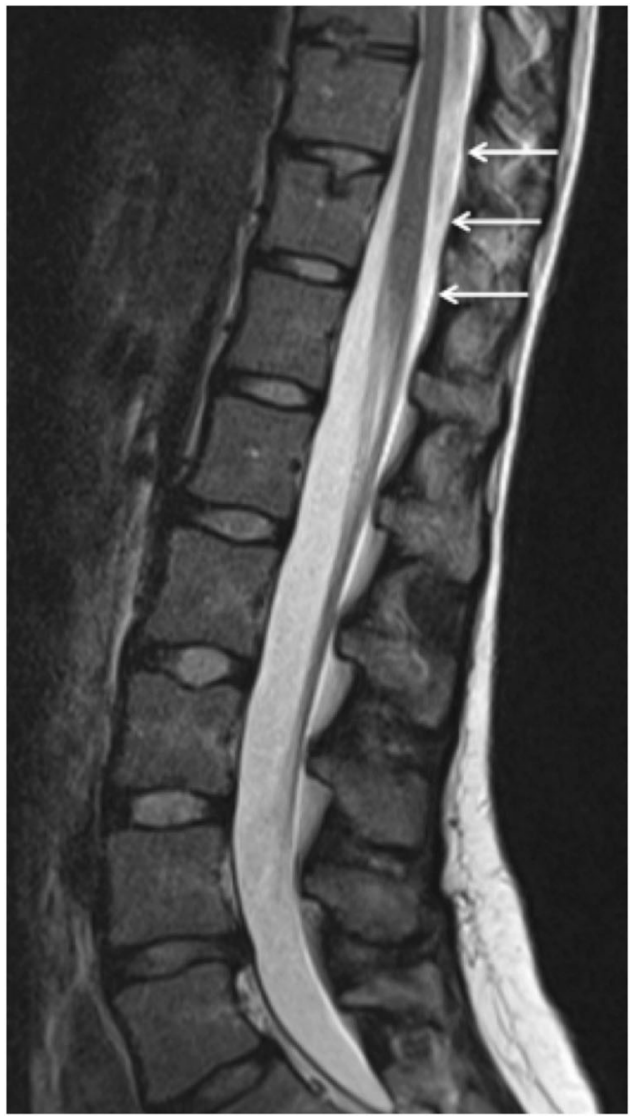

Fig. 1 Sagittal T2-weighted image of the lumbar spine illustrates diffuse intramedullary high signal intensity signal with minor expansion of the conus such as alcohol [1]. The purpose and expected duration of the block will determine which agent to select [1].

The use of particulate steroids for spinal injections is controversial [15]. A number of recent publications have reported that epidural steroid injections using particulate steroid at the level of cervical, lumbar, and lumbosacral spine may be associated with a risk of infarction ranging from the brainstem to the distal spinal cord $[5,6,15]$. Some authors speculate that the reasons for such adverse event could include formation of thrombus, vasospasm, or dissection causing vascular injury $[6,15]$.

Up till 2015, all cases of spinal cord injury described in the literature involved injections of particulate steroids into the epidural space [15]. Triamcinolone acetonide, as used in this case, is a particulate steroid among other examples like methylprednisolone acetate and prednisolone acetate [15]. These are poorly water-soluble and can cause crystallised precipitants ranging from 0.5 to over $1000 \mu \mathrm{m}$ in hydrophilic solutions, subsequently risking small arterial vessel occlusions following incidental steroid injection into an artery during procedures [15]. There has been one reported case by Gharibo et al. [6] of conus infarction after a right L4 transforaminal epidural steroid injection using nonparticulate steroid dexamethasone in 2016. Kenalog-40 (triamcinolone acetonide injectable suspension, USP) has only been approved by FDA for intramuscular and intraarticular use and is not licensed for epidural use due to reported cases of serious adverse neurological events, including spinal cord infarction, paraplegia, brainstem stroke, and even deaths [16].

In 2017, the first case of unusual neurological complication post-GIB was reported by Gupta et al. [7] as a
Fig. 2 a, b Sequential axial T2weighted image through the conus medullaris demonstrates intramedullary centrally located high signal
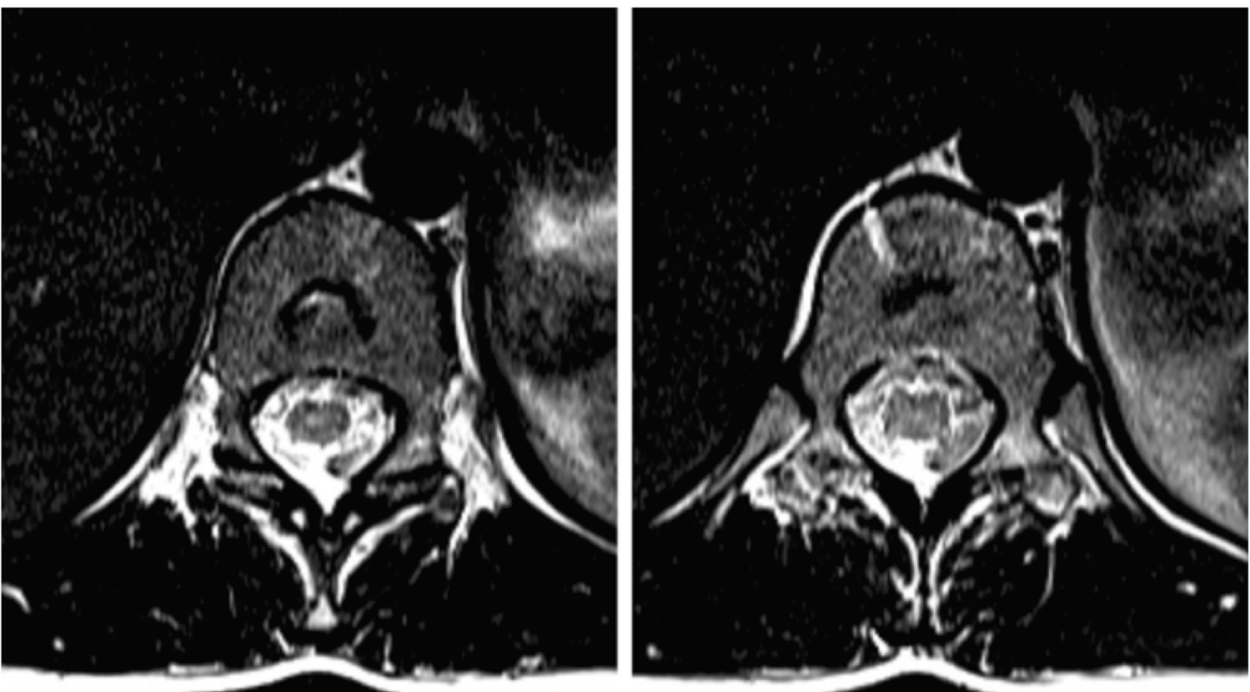

a

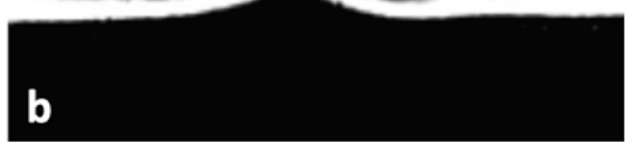




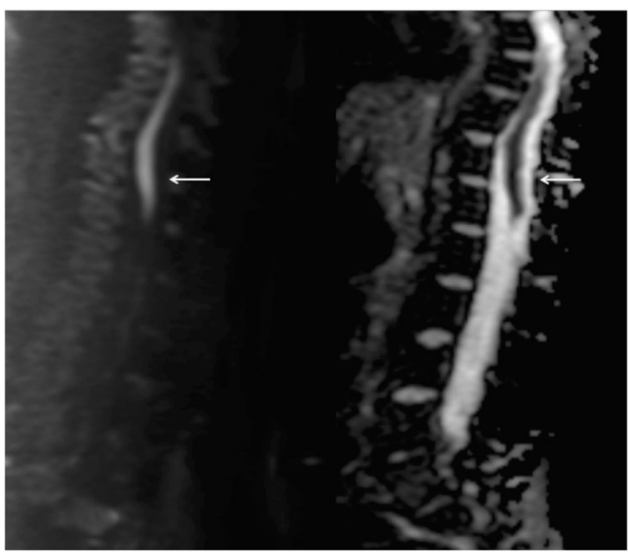

Fig. 3 Diffusion-weighted image and apparent diffusion coefficient demonstrates hyperintense DWI signal within the conus and corresponding hypointense signal on ADC. The appearances are consistent with restricted diffusion within the conus

sudden-onset right thigh pain likely due to a delayed response of the alcohol in the block, followed by right lower limb movement difficulties with loss of bladder and bowel control $3 \mathrm{~h}$ after the procedure. This patient had $50 \%$ loss of sensation from L3 dermatome downwards with an antalgic gait. Forty-eight hours after treatment with dexamethasone and morphine, his bladder and bowel function improved with a restored normal sensation and gait [7].

Our case is the first reported case of imaging-documented conus infarction post-GIB, further demonstrating the risk of rare neurological complications after GIB. Symptoms suggestive of a suspected disturbance to the conus medullaris or nerve roots include back pain, leg weakness, sensory loss, scoliosis, foot deformities, bladder, or rectal dysfunction [5]. While the mechanism by which this patient's neurological complications occurred cannot be definitely established, the development of neurological disturbance immediately following unguided injection of particulate steroid would strongly imply a causal relationship. We speculate that this case may be related to inadvertent intravascular injection of local anaesthetic and particulate steroid into one of the vessels in the presacral plexus during GIB. Both thromboembolism and direct oppression caused by foreign injectates in spinal cord arteries can lead to spinal cord infarction [17]. Typically, conus medullaris infarction presents with mainly lower motor neuron findings and has neural segments from S2 and below. However, conus lesions can be complicated and have patchy distributions or epiconus involvement. In our case the patient had upper motor neuron findings and neurological symptoms extending to the L2-3 myotomes. This suggests that while the detectable signal abnormality on acute MRI was limited to the conus, the range of neurological injury may have been more extensive with potential involvement of the cauda equina. We note that Gupa et al. [7] also reported sensory disturbance extending to the L3 dermatome in their case of transient neurological complications following GIB. As this was an unguided injection, the exact location of needle placement prior to injection is unknown. Depending on the angle and length of the needle, it is even possible to have injected via the caudal canal, which may then have resulted in inadvertent epidural delivery. However, how this would result in permanent neurological injury is unclear.

Possible preventative safety measures include performing MRI before GIB to identify variant anatomy, using image guidance during GIB, and aspirating to check for blood or cerebrospinal fluid in the needle prior to injection. Non-particulate steroid such as dexamethasone has been associated with fewer neurological complications than particulate steroid solutions and may be a safer option than particulate steroids for these procedures. We would suggest that imaging guidance with CT or fluoroscopic control and injection of contrast material should be considered when performing such procedures to minimise the risk of inadvertent intravascular injection.

\section{Conclusion}

We present the first reported case of imaging-documented conus medullaris infarction after a conventional non-imaging guided transsacrococcygeal ganglion impar block for the management of coccydynia using particulate steroid solution. Care must be taken to minimise the risk of inadvertent intravascular injection of particulate steroids when doing such procedures, and we would advocate use of imaging guidance with use of contrast to minimise risk of inadvertent vascular injection. Clinicians must have a high index of suspicion for such complications if patients develop neurological symptoms following ganglion impar injections.

\section{Compliance with ethical standards}

Conflict of interest The authors declare that they have no conflict of interest.

Publisher's note Springer Nature remains neutral with regard to jurisdictional claims in published maps and institutional affiliations.

\section{References}

1. Gunduz $\mathrm{OH}$, Kenis-Coskun O. Ganglion blocks as a treatment of pain: current perspectives. J Pain Res. 2017;10:2815-26.

2. Oh C-S, Chung I-H, Ji H-J, Yoon D-M. Clinical implications of topographic anatomy on the ganglion impar. Anesthesiology. 2004;101:249-50.

3. Toshniwal GR, Dureja GP, Prashanth SM. Transsacrococcygeal approach to ganglion impar block for management of chronic perineal pain: a prospective observational study. Pain Physician. 2007;10:661-6. 
4. Walters A, Muhleman M, Osiro S, Bubb K, Snosek M, Shoja $\mathrm{MM}$, et al. One is the loneliest number: A review of the ganglion impar and its relation to pelvic pain syndromes. Clin Anat. 2013;26:855-61.

5. Wang G, Liang J, Jia Z, Wan L, Yang M. Spinal cord infarction caused by sacral canal epidural steroid injection. Medicine (U S). 2018;97:E0111.

6. Gharibo CG, Fakhry M, Diwan S, Kaye AD. Conus medullaris infarction after a right 14 transforaminal epidural steroid injection using dexamethasone. Pain Physician. 2016;19: E1211-4.

7. Gupta N, Garg R, Saini S, Bharti SJ, Kumar V. An unusual complication after ganglion impar block for chronic cancer pain management. AANA J. 2017;85:424-6.

8. Foye PM, Buttaci CJ, Stitik TP, Yonclas PP. Successful injection for coccyx pain. Am J Phys Med Rehabil. 2006;85: 783-4.

9. Plancarte DR, Amescua C, Patt RB, Allende S. Presacral blockade of the ganglion of walther (ganglion Impar). Anesthesiol $\mathrm{J} \mathrm{Am}$ Soc Anesthesiol. 1990;73(3A):A751.

10. Huang JJ. Another modified approach to the ganglion of Walther block (ganglion of impar). J Clin Anesth. 2003;15: 282-3.
11. Lin CS, Cheng JK, Hsu YW, Chen CC, Lao HC, Huang CJ, et al. Ultrasound-guided ganglion impar block: A technical report. Pain Med. 2010;11:390-4.

12. Datir A, Connell D. CT-guided injection for ganglion impar blockade: a radiological approach to the management of coccydynia. Clin Radio. 2010;65:21-5.

13. Marker DR, U-Thainual P, Ungi T, Flammang AJ, Fichtinger G, Iordachita II, et al. MR-guided perineural injection of the ganglion impar: technical considerations and feasibility. Skelet Radio. 2016;45:591-7.

14. Scott-Warren JT, Hill V, Rajasekaran A. Ganglion impar blockade: a review. Curr Pain Headache Rep. 2013;17:306.

15. Dietrich TJ, Sutter R, Froehlich JM, Pfirrmann CWA. Particulate versus non-particulate steroids for lumbar transforaminal or interlaminar epidural steroid injections: an update. Skelet Radio. 2015;44:149-55.

16. KENALOG ${ }^{\circledR}-40$ INJECTION (triamcinolone acetonide injectable suspension, USP) [Internet]. [cited 22 May 2019]. Available from: https://dailymed.nlm.nih.gov/dailymed/fda/fdaDrugXsl.cfm? setid=e25cfc22-29ba-473f-88c4-3c88e9f6863b\&type=display.

17. Ludwig MA, Burns SP. Spinal cord infarction following cervical transforaminal epidural injection. Spine (Philos Pa 1976). 2005;30:E266-8. 